\title{
Untersuchungen über die pH-Abhängigkeit, die Hemmbarkeit und Reaktivierbarkeit von Angiotensin II und Angiotensin II-amid spaltenden Enzymen des menschlichen Plasmas')
}

\author{
Von M. Zwanzig und W. Oelkers \\ Aus der II. Medizinischen Klinik und Poliklinik Berlin (Direktor: Prof. Dr. M. Schwab)
}

(Eingegangen am 17. Dezember 1968)

\begin{abstract}
Die pH-Abhängigkeit dex biologischen Inaktivierung von $\alpha$-L-Aspartyl1-Angiotensin $U(A)$ und von $\alpha$-L-Asparaginyl ${ }^{1}$-Angiotensin II (H) durch menschliches Plasma sowie die pH-Abhängigkeit det Hemmbarkeit von „Angiotensinasen“ durch EDTA und ihre Reaktivierung durch zweiwertige Metallionen wurden vergleichend untersucht. Beide Substrate werden durch mindestens drei in verschiedenen $\mathrm{pH}-$ Bereichen optimal wirksame Enzyme gespalten. In allen pH-Bereichen fanden sich unterschiedliche Eigenschaften der A- bzw. H-iniktivierenden Enzyme. Zusätzlich wurde die pH-Abhängigkeit der biologischen Inaktivierung von $\mathrm{H}$ und der Abspaltung von Asparagin us $\mathrm{H}$ (Aminopeptidase) verglichen. Die Annahme, daß die bei pH 5,6 optimal wirksamen Enzyme Endopeptidasen und die im Neutralsereich und im Alkalischen optimal wirksamen Enzyme Aminopeptidasen sind, ist mit unseren Ergebnissen vereinbar.
\end{abstract}

Studies on the pH-dependence, inbibition and reactivation of angiotensin $I I$ and angiotensin II-amide splitting env'mes in buman plasma

We compared the $\mathrm{pH}$-dependance of the inactivation of $\alpha$-L-asparty ${ }^{1}$-angiotensin $I I(A)$ and of $\alpha$-L-asparaginyli-angiotensin II (H) by juman plasma. In addition, the pH-dependance of EDTA-inhibition of "angiotensinases" and its reversal by divalent cations were inrestigated. Both substrats are split by at least three enzymes with different $\mathrm{pH}$-optima. There are differences between the $\mathrm{A}$-and $\mathrm{H}$-splitting enzymes in the acid, neutral and alkaline range. We further investigated the $\mathrm{pH}$-dependance of the hydrolysis of the $\mathrm{N}$-terminal peptide bond in $\mathrm{H}$. Our results are compatible with the assumption that the enzymes most active at $\mathrm{pH} 5.6$ are endopeptidases, while the enzymes with $\mathrm{pH}$-optima in the neutral and alkaline range are aminopeptidases.

SEGOLI und Mitarbeiter (1) äußerten als erste die Vermutung, daB Asparaginyl1 ${ }^{1}$-Angiotensin $I I(H)$ und Aspartyl1-Angiotensin II (A) im Blutplasma durch unterschiedliche Enzyme inaktiviert werden. NaGatsu und Mitarbeiter (2) konnten diese Annahme weitgehend bestätigen. Sie fanden, da $B$ die Hydrolyse von $\alpha$-IAspartyl ${ }^{1}$ - bzw. $\alpha$-L-Glutamyl- $\beta$-Naphthylamid durch $A$, nicht aber durch $\mathrm{H}$ kompetitiv gehemmt wird. Die Hydrolyse von L-Leucyl- $\beta$-Naphthylamid wird andererseits nur durch $\mathrm{H}$ kompetitiv gehemmt. Während die Hydrolyse der N-terminalen Peptidbindung von $\mathrm{A}$ in unverdünntem Serum bei $\mathrm{pH} 7,0$ durch $12 \mathrm{mMCa}^{++}$ aktiviert und durch $2,4 \mathrm{~mm} \mathrm{Ni} i^{++}$vollständig gehemmt wird, hemmt $\mathrm{Ca}^{++}$die entsprechende Reaktion bei der Hydrolyse von $\mathrm{H}$ schwach und $\mathrm{Ni}^{++}$nur um $50 \%$. Âhnliche Unterschiede ergaben sich bei der Untersuchung der Hydrolyse von $\alpha$-L-Aspartyl- $\beta$-Naphthylamid bzw. L-Leucyl- $\beta$-Naphthylamid in Gegenwart von $\mathrm{Ca}^{++}$ und $\mathrm{Ni}^{++}$(3). Die Autoren betonen die Ähnlichkeit der Hemmungs- und Aktivierungscharakteristik des enzymatischen Abbaues von $\mathrm{A}$ im Plasma mit der eines von GlenNer und McMillan (4) in Rattennieren gefundenen Enzyms (Aminopeptidase A), das spezifisch $N$-terminale $\alpha$-L-Aspartyl- und $\alpha$-L-Glutamyl-Peptidbindungen spaltet. Für die Hydrolyse von $\mathrm{H}$ dagegen werden mehrere weniger spezifische Peptidasen angenommen, die zum Teil auch N-terminale L-LeucylPeptidbindungen spalten.

1) Der Inbalt dieser Arbeit wurde auszugsweise auf der 74. Tagung der Deutschen Gesellschaft für Innere Medizin in Wiesbaden (22.-25. 4. 1968) vorgetragen.
Die von Khatrallah und Mitarbeitern (5) beschrie-

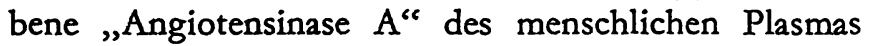
soll spezifisch $\mathrm{N}$-terminale $\alpha$-L-Aspyrtyl- und $\alpha$-LAsparaginyl-Peptidbindungen spalten. AngiotensinAnaloge wie $\beta$-Aspartyl1-Angiotensin II und Argingl ${ }^{1-}$ Angiotensin $\Pi$ werden nicht angegriffen. Khairallah und Mitarbeiter (6) konnten aber spāter durch Ammoniumsulfat-Fraktionierung die $\alpha$-L-Aspartyl-Peptidespaltende Enzymaktivităt von der $\alpha$-L-AsparaginylPeptide-spaltenden trennen. Die zum Teil widersprüchlichen Angaben in der Literatur über Substratspezifität, Hemmbarkeit und Reaktivierbarkeit Angiotensin-spaltender Enzyme im Plasma beginnen sich allmählich zu entwirren.

Wir haben in der vorstehenden Mitteilung ( 7 ) bereits darauf hingewiesen, da $\beta$ die Bedeutung der Plasmaangiotensinasen (ATasen) für die Begrenzung der biologischen Wirksamkeit von Angiotensin wahrscheinlich gering ist. Wichtig ist die Kenntnis ihrer Eigenschaften aber bei in-vitro-Versuchen, zum Beispiel bei der Reninbestimmung. Die ATasen müssen inaktiviert werden, damit das bei der enzymatischen Reaktion zwischen Renin und Angiotensinogen (Reninsubstrat) gebildete Angiotensin nicht zerstört wird und quantitativ bestimmt werden kann. $\mathrm{Da}$ bei manchen Methoden der Reninbestimmung die Renin-Substratreaktion nicht im Neutralbereich stattfindet $(8,9,10)$, ist auch die Kenntnis der im schwach Saueren wirksamen Angiotensinspaltenden Enzyme wichtig.

Wir berichten im Folgenden über vergleichende Untersuchungen der biologischen Inaktivierung von $\mathrm{A}$ und $\mathrm{H}$ 
in Abhāngigkeit ron der Wasserstoffionen-Konzentration im menschlichen Plasma. Daneben wurde die Abhängigkeit der Inakirierbarkeit von ATasen durch den Cbelatbildner Athỵlendiamintetraessigsāure (EDTA) und ihrer Reaktivierbarkeit durch zweiwertige Metallionen ron der IVasserstoffionenkonzentration untersucht. $\mathrm{Da}$ uns der Vergleich der Substrare $A$ und $\mathrm{H}$ wichtig erschien, konnten wir den von uns beschriebenen optischen Test (11) zur Bestimmung der AngiotensinAminopeptidase-Altivitāt nicht verwenden. Die dafür erforderlichen großen Mengen Aspartyl-Angiotensin II standen nicht zur Verfügung. Wir bedienten uns deshalb eines bei rielen früheren Untersuchungen $(1,5,6,12)$ angewandten Verfahrens. Der Verlust der rasopressorischen Wirkung einer bekannten Menge Angiotensin nach Inkubation mir Plasma unter definierten Bediagungen wird an der narkotisierten Ratte gemessen. Mlit dieser Methode können nur relative Enzrmaktivitäten bestimmt werden, da keine Substratsāttigung der Enzyme gegeben ist und da sich die Reaktionsgeschwindigkeit durch Abnahme der Substratkonzentration während der Inkubation stāndig verringert. Die Methode genügt aber dem Anliegen dieser Lintersuchung und ist hinreichend genau.

\section{Methodik}

\section{Cintersucbumgsmaterial}

Die Untersuchungen wurden mit Heparinplasma von gesunden Versuchspersonen (2.000 E Heparin $/ 100 \mathrm{~m} /$ Vollblut) durchgeführ Das Plasma wurde bis zur weireren Verwendung bei $-20^{*}$ gefroren aufbewahrr.

Inksbation ron Plasma mit Angiotensin zur Bestimnnsung seiner biologiscben Inaktivierung

Die Inkubacionsansātze enthielten:

$1,0 \mathrm{~m} /$ Substratlösung [ $500 \mathrm{ng} x-\mathrm{L}$-Aspart ${ }^{1}$-Angiotensin $I I \mathrm{bzw}$. $500 \mathrm{ng}$ - -L-Asparaginy.l'1-Angiotensin II (Hypertensin CIBA), gelöst in $1 \mathrm{~m} / 0,05 \mathrm{~N}$ Tris-Mlaleatpuffer unterschiedlicher $\mathrm{pH}$ Trerte].

$3,9 \mathrm{~m} / 0,05 \mathrm{~N}$ Trismaleatpuffer des gleichen $\mathrm{pH}-\pi$ Tertes. Der Trismaleatpuffer wurde nach den Angaben der Wissenschaftlichen Tabellen Documenca Geigy 1262, Seite $2 i T$ und 2i8, hergestellt. Für die Bestimmung der $\mathrm{pH}$-Abhängigkeir der biologischen Inaktirierung ron $A$ und $H$ wurden Abstände von $0,2-0,4$ pH-Einheiten zwischen $\mathrm{pH} \mathrm{5,2}$ und $\mathrm{pH}$ S,6 gewählt. Für die Reaktivierungsversuche mit unterschiedlichen Metallionenkonzentrationen wurden jewreils drei $\mathrm{pH}-\mathrm{W}$ 'erte gewählt, die den rorher ermittelten $\mathrm{pH}$-Optima der biologischen Inaktirierung entsprachen.

$0, \overline{\mathrm{m}} / 0,9$ proz. $\mathrm{NaCl}-\mathrm{Lösung}$ für die Bestimmung der $\mathrm{pH}-\mathrm{Op}$ timum-Kurren beider Substrate. Bei Inkubation mit EDTA oder Metallsalzen wurde das Zehnfache der gewrünschten Endkonzentration von $\mathrm{Na}_{2}$-Athylendiamintetraessigsäure (Titriplex III der Firma Merck) oder der Metall-Dichloride oder beider zusammen in $0,5 \mathrm{ml}$ 0,9proz. $\mathrm{NaCl}$-Lösung hinzugefügt. Die Lösungen wurden vorher mit $\mathrm{NaOH}$ auf $\mathrm{pH} 7,0$ eingestellt.

$0,1 \mathrm{~m} /$ einer 5proz. wäßr. Lösung von Neomycinsulfat (Bykomycin der Firma BYK-Gulden) zur Verhinderung von Bakterienwachstum.

$0,5 \mathrm{~m} /$ Mischplasma, dessen $\mathrm{pH}$ auf 7,0 eingestellt wurde.

Die Ansātze wurden im Eisbad hergestellt. Unmittelbar nach der Zugabe des Plasmas und gründlichem Mlischen wurden $2 \mathrm{~m} /$ als Leerwert (nichrinkubierter Ansatz) entnommen und in ver- schlossenen Glasröhrchen $10 \mathrm{Min}$. im Wasserbad auf $100^{\circ}$ erhitzt. Die koagulierten Proteine wurden mit 3000 U. Min. abzentrifugiert und der Uberstand in Polystyrol-Röhrchen bei $-20^{\circ}$ bis zur Testung an der Ratte eingefroren. Der verbleibende Ansatz (3 m/) wurde, wenn $H$ das Substrat war, 10 Min., wenn A das Substrat war, $120 \mathrm{Mlia}$. bei $37^{2}$ inkubiert. Nach Beendigung der Inkubacion wurden die Ansäze sofort auf $100^{\circ}$ exhitzt und weiter wie die Leerwerte behandelt.

Nach Feststellung der pH-Optima der Angiotensin-Inaktivierung durch menschliches Plasma ururde die Hemmbarkeit der ATasen durch Dialyse gegen 0,9proz. $\mathrm{NaCl}-L$ ösung bzw. gegen $\mathrm{Na}_{2}-$ EDTA in 0,9proz. NaCl-Lösung geprüft.

Je $50 \mathrm{~m} /$ Plasma wurden a) sofort eingefroren, b) 9 Tage bei $3^{\circ}$ gegen 0,9proz. $\mathrm{NaCl}$-Lösung dialysiert, c) 5 Tage bei $3^{\circ}$ gegen $10 \mathrm{mv}$ bzr. 1,0 mv Na $\mathrm{Na}_{2}$-EDTA in 0,9proz. NaCl-Lösung (auf pH 7,0 eingestellt), anschließend 4 Tage gegen 0,9 proz. $\mathrm{NaCl}$ Lösung dialrsiert. Die Dialysierflüssigkeit wurde täglich gewechselt. Nach Beendigung der Dialyse wurden Volumendifferenzen der Plasmaproben durch Zugabe ron 0,9proz. NaCl-Lösung ausgeglichen.

Dann wurde die biologische Inaktivienung ron $A$ und $H$ durch unbehandeltes bzw. dialysiertes Plasma geprüft Inkubacionsansatz siehe oben). A wurde mir Plasma bei pH 5,6,6,8 und 8,0, $\mathrm{H}$ bei pH 5,6,7,2 und 8,6 inkubiert. Die Inkubationszeiren wurden so gewählt, daß bei jedem $\mathrm{pH}$-Wert durch unbehandeltes Plasma etra 50,'o der Substrate A oder $H$ inaktiviert wurden (s. Tab. 1). Danach wurde die Reaktivierbarkeit der durch Dialyse gegen EDTA gehemmten ATasen geprüft. Die Ansätze enthielten rerschiedene Konzentrationen $\mathrm{CaCl}_{2}, \mathrm{MgCl}_{2}, \mathrm{ZnCl}_{2}, \mathrm{MnCl}_{3}$ und $\mathrm{CoCl}_{\mathbf{g}}$.

\section{Angiotensinbestinsmung an der Ratte}

Männliche Wistar-Ratten (200-300 g) wurden in Pentobarbital narkose nephrektomiert. $24 \mathrm{Stdn}$. später wurden sie in Urethannarkose (2 m/20proz. Urethanlösung i. m.) tracheotomiert. In die rechre Vena jugularis und in die linke Arteria carotis murden Polyäthṛlenkatheter eingeführt. Der Druck in der Arteria carotis wurde über ein $\mathrm{Hg}$-Manometer mir Tintenschreiber am Krmographen registriert. In die Jugularrene nurden $0,2 \mathrm{~m} /$ der $z u$ restenden Lösung bzw. $0,2 \mathrm{~m} /$ der Angiotensin-Standardlösung injiziert. Danach wurde $0,1 \mathrm{~m} /$ Ringer-Lösung injiziert. Die Angiotensin-Standardlösungen enthielten $50 \mathrm{ng}$ bztr. $100 \mathrm{ng}$ Hypertensin CIBA in $1 \mathrm{~m} /$ Ringer-Lösung. Jede unbekannte Probe, auch die Leenrerte, wurden zweimal mit dem unmittelbar vorher und nachher injizierten Hypertensin-Standard verglichen. Alle angegebenen Inaktivierungsraten sind Mitteltrerte ztreier Bestimmungen. Die biologische Inaktivierung wird als prozentualer Verlust der pressorischen Wirkung des inkubierten Ansatzes im Vergleich mit dem nicht inkubierten Ansaz angegeben. Eine Spontanhydrolyse ron Angiotensin wurde in Ansätzen, die statt des Plasmas 0,9proz. NaCl-Lösung enthielten, bei keinem der geprüften $\mathrm{pH}$-Werte zwischen 5,2 und 8,6 festgestellt.

Die Angiotensinbestimmung mit dem angegebenen Verfahren ist gut reproduzierbar. Die Differenzen der Inaktirierungsraten zweier Bestimmungen der gleichen Probe an ztrei verschiedenen Ratten betrugen bei 31 willkürlich ausgewählten Doppelbe stimmungen im Mittel 3,9\% $\left(0_{i 0}^{\circ}\right.$ bis $\left.10^{\circ},{ }^{\circ}\right)$. Die Standardabweichung ist $3,1^{\circ} ;$. Inaktivierungsraten über $10 \% \%$ bztr. Differenzen zwischen verschiedenen Inaktivierungsraten über $10 \%$ sind daher als signifikant anzusehen.

Messumg der Asparagin-Abspaltumg aus a-L-Asparaging/ ${ }^{-}$-Angiotensin II Aminopeptidase-Aktivität

Das Prinzip der von uns entwickelten Methode wurde in der vorstehenden Mlitteilung (i) angegeben und ist bei (11) genauer beschrieben. Zum Vergleich der $\mathrm{pH}$-Abhängigkeit der Aminopeptidase-Aktivităt des Plasmas gegenüber $\mathrm{H}$ mit der $\mathrm{pH}$-Abhängigkeit der biologischen Inaktivierung ron $\mathrm{H}$ wurde in $0,05 \mathrm{~N}$ Trismaleat-

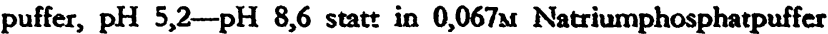
(11) 60 Mlin. inkubiert. Die Aminopeptidase-Aktivität (Abb. 2) ist hier nicht in IE/l, sondern in Extinktions-Einheiten angegeben. 


\section{Ergebnisse}

Vergleich der $p H$-Abbängigkeit der biologischen Inaktivierung von $A$ und $H$ durch menscblicbes Plasma (Abb. 1)

Für eine vergleichbare Inaktivierung muß $A$ etwa 10 mal länger mit dem verdünnten Plasma inkubiert werden als $\mathrm{H}$. Die Optima der Inaktivierung von A

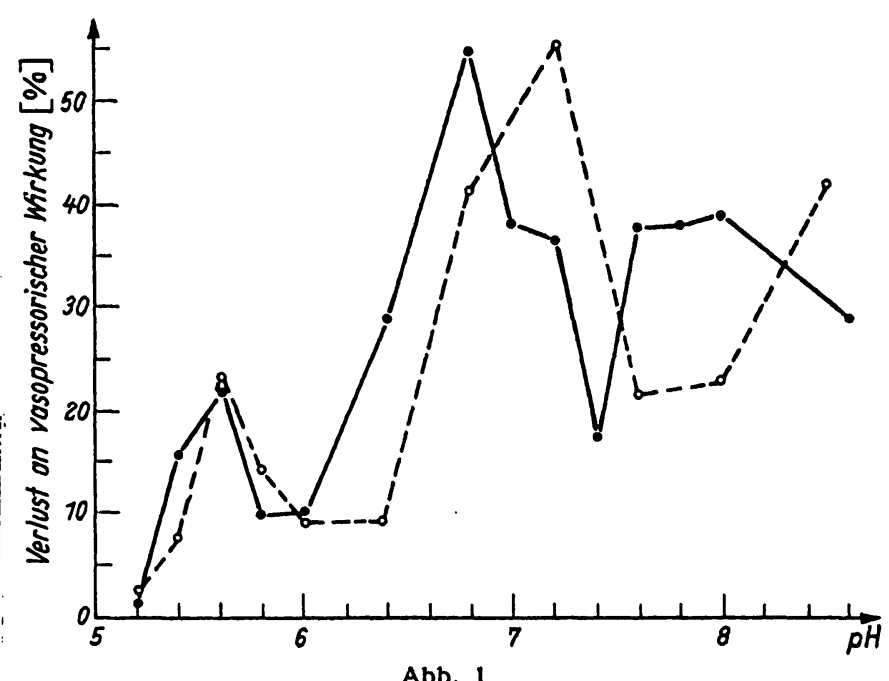

Vergleich der biologischen Inaktivierung von A $(\bullet-\bullet)$ und H (o- - - durch 1:10 verdunntes Plasma in Abhangigkeit vom pH des 0,04M Trismaleatpuffers. Inkubationsdauer mit Substrat
H: 10 Min., mit Substrat A: 120 Min. Jeder Punkt entspricht einem

liegen bei $\mathrm{pH} 5,6, \mathrm{pH} 6,8$ und $\mathrm{pH} 7,6-8,0$. Bei $\mathrm{pH}$ 6,8 ist die Inaktivierung absolut am größten. Die Optima der Inaktivierung von $\mathrm{H}$ licgen bei $\mathrm{pH} 5,6$ und $\mathrm{pH} 7$,2. Jenseits $\mathrm{pH} \mathrm{8,0}$ wird eine weitere Zunahme der Inaktivierung von $\mathrm{H}$ beobachtet, deren Maximum in Trismaleatpuffer nicht ermittelt werden konnte. Die $\mathrm{pH}-$ Optima der Inaktivierung von $\mathrm{A}$ und $\mathrm{H}$ stimmen also im Sauren überein, während sich im Neutralbereich und im Alkalischen Unterschiede finden.

Vergleich der pH-Abbängigkeit der biologiscben Inaktivierung von $H$ mit der Spaltung von $H$ durcb Aminopeptidasen (Abb. 2)

In Ubereinstimmung mit früheren Ergebnissen der Bestimmung des $\mathrm{pH}$-Optimum in Acetat- und Phosphat-

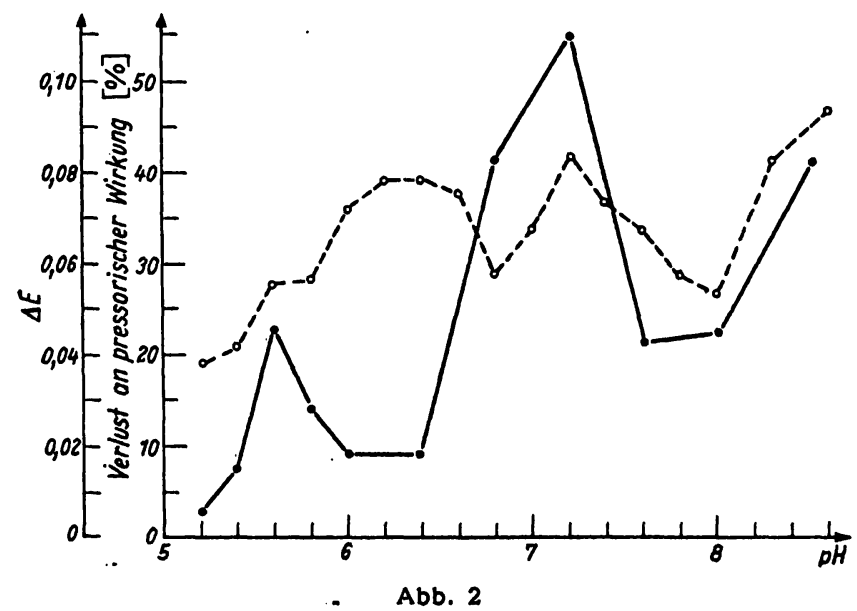

Vergleich der biologischen Inaktivierung (\% Verlust an pressorischer Wirkung) von $H_{(-1}$ mit der Geschwindigkelt der AsparaginAbspaltung aus $H(\circ-\overrightarrow{-}-\rightarrow$ ) in Abhängigkeit vom $\mathrm{pH}$ des $0,04 \mathrm{M}$ Trismaleatpuifers. Die Aminopeptidase-Aktivitat (Mittelwerte von
zwei Inkubationsserien) ist in Extinktionseinheiten $(366 \mathrm{~nm})$ angegeben puffer (11) fanden wir ein pH-Optimum der Aminopeptidase-Aktivität gegenüber $\mathrm{H}$ bei $\mathrm{pH}$ 6,2-6,4. Ein weitcres, nicht scharf gegen das vorige abgehobenes Optimum fanden wir in Ubercinstimmung mit $\mathrm{dcm}$ Optimum der biologischen Inaktivierung von $\mathrm{H}$ bei $\mathrm{pH}$ 7,4. Jenseits $\mathrm{pH} 8,0$ nimmt die AminopeptidaseAktivität gegenüber $H$, ähnlich wic die biologische Inaktivierung, wieder zu.

Die Beeinflussung der biologischen Inaktivierung won $A$ und $H$ durcls $\mathrm{Ca}^{++}$und $\mathrm{Na}_{2} E D T A$ in Abbängigkeit voin $\mathrm{pH}$ (Abb. 3 und 4)

$\mathrm{Na}_{2}$ ED'TA (1 mM) hemmt die biologische Inaktivierung von $A$ und $\mathrm{H}$ im Sauren (pH-Optimum 5,6) nahezu vollständig. Calcium, das in $1 \mathrm{~mm}$ Konzentration allein zugesetzt, den Abbau von $\mathrm{A}$ und $\mathrm{H}$ nicht signifikant verändert, hebt die ED'TA-Hemmung bei $\mathrm{Zu}$ gabe eines zweifachen Uberschusses $\left(3 \mathrm{~mm} \mathrm{Ca}{ }^{++}\right)$nicht auf. $/$ Die geringfügige Inaktivierung von $\mathrm{A}$ und $\mathrm{H}$ um etwa $5 \%$ bei pH 5,6 in Gegenwart von $\mathrm{Ca}^{++}$und EDTA ist nicht signifikant. Im Neutralbereich hemmen $1 \mathrm{~mm}$

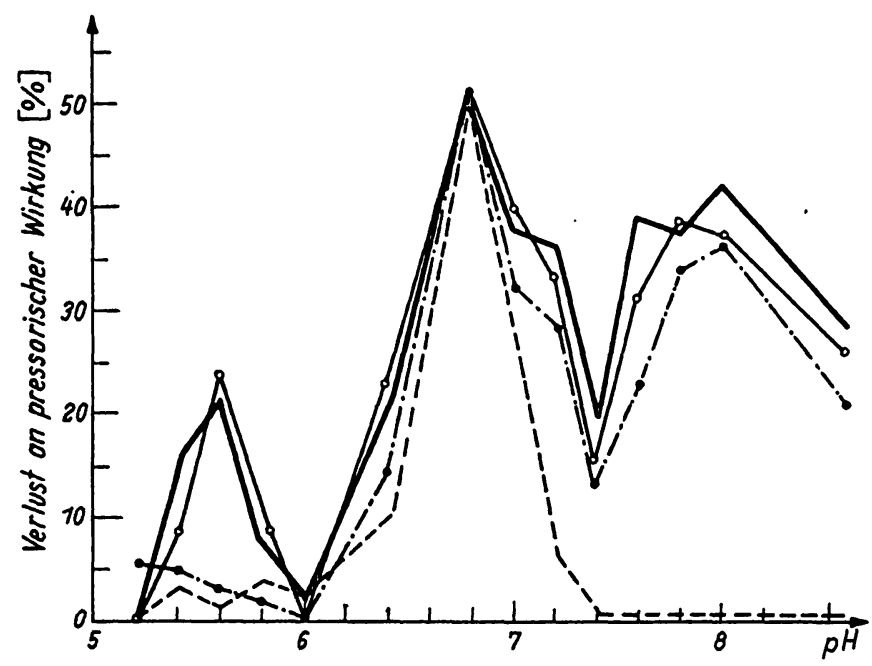

Abb. 3

Biologische Inaktivierung von $A$ durch $1: 10$ verdünntes menschliches Plasma in Abliangigkeit vom pH des 0,04n Trismaleatpuffers ohne Zusatz von Affektoren ( $\longrightarrow$ ), mit I muNa, EDTA (- - ), mit

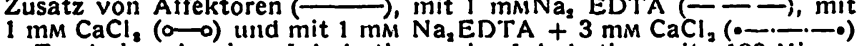

$\underset{\text { Ergebnisse je einer Inkubationsserie. Inkubationszeit: }}{\mathrm{mM} \mathrm{CaCl}_{2}(0 \mathrm{Min} \text {. }}$

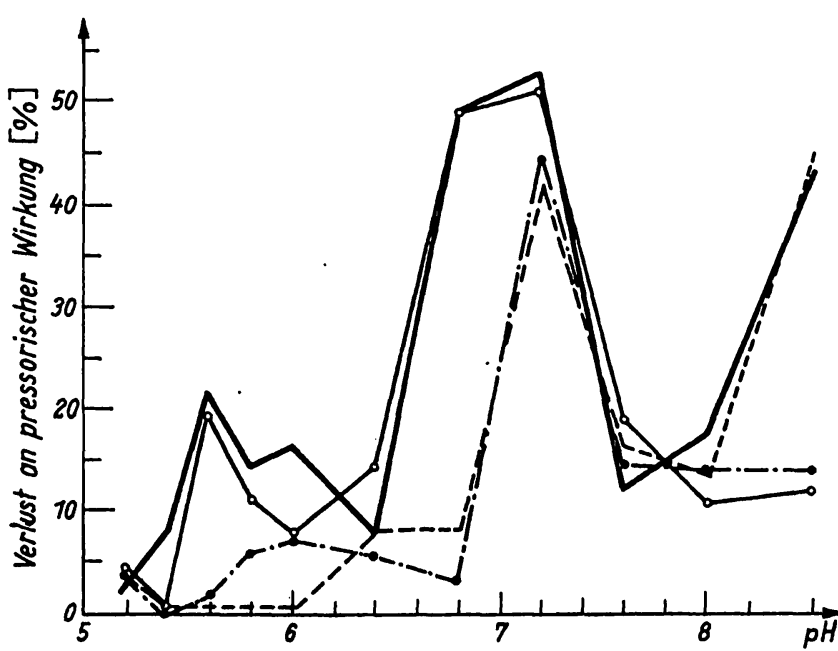

Abb. 4

Blologische Inaktivierung von $H$ durch $1:$ ! 0 verdïmitesmenschliches Plasmia mit und ohne Affektoren. Zeichenerkläruing sieheAbbildung 3. Inkubationszelt: $10 \mathrm{Min}$. 
EDTA die biologische Inaktivierung von $\mathrm{A}$ und $\mathrm{H}$ nicht signifikant. Auch $\mathrm{Ca}^{++}$allein und EDTA $+\mathrm{Ca}^{++}$ beeinflussen die Inaktivierung von $\mathrm{A}$ und $\mathrm{H}$ nicht bemerkenswert.

Deutliche Unterschiede zwischen $\mathrm{A}$ und $\mathrm{H}$ finden sich im Alkalischen. EDTA (1 mMr) hemmt die Inaktivierung von $\mathrm{A}$ vollständig. $\mathrm{Ca}^{++}$(bei $1 \mathrm{~mm}$ Konzentration allein wirkungslos) hebt die EDTA-Hemmung mit $3 \mathrm{mis}$ Konzentration vollständig auf. Die biologische Inaktivierung von $\mathrm{H}$ wird erst $\mathrm{ab} \mathrm{pH} 8,0$ durch EDTA teilweise gehemmt. $\mathrm{Ca}^{++}$hebt die EDTA-Hemmung nicht auf.

Hemmung der biologischen Inaktivierung von $A$ und $H$ durch Dialj'se von Plasma gegen 0,9proz. Kocbsalzlösung und EDTA-Lösung und Reaktivierung durch queinertige Metallionen (Tab. 1)

Dialyse von Plasma gegen 0,9proz. NaCl-Lösung hemmt die biologische Inaktivierung von $\mathrm{A}$ und $\mathrm{H}$ im Sauren vollständig. Im Neutralen und im Alkalischen wird die biologische Inaktivierung von $\mathrm{A}$ und $\mathrm{H}$ partiell gehemmt.

$10 \mathrm{mM}$ EDTA hemmt die biologische Inaktivierung von $A$ in allen pH-Bereichen vollständig. Die Inaktivierung von $\mathrm{H}$ wird im Sauren total, im neutralen und im alkalischen Bereich subtotal gehemmt.

$\mathrm{Ca}^{++}(2,5 \mathrm{~mm})$ hebt die Hemmung der biologischen Inaktivierung von $\mathrm{A}$ durch EDTA-Dialyse in allen $\mathrm{pH}-B$ ereichen auf. $0,25 \mathrm{mmCa}^{++}$reaktiviert bei $\mathrm{pH} 6,8$

Tab. 1

Biologische Inaktivierung von $\mathrm{A}$ und $\mathrm{H}$ bei verschiedenen $\mathrm{pH}-$ Werten nach Dialyse gegen 0,9proz. NaCl-Lösung bzw. gegen $10 \mathrm{mMNa}_{2}$ EDTA-Lösung. Reaktivierbarkeit der Angiotensin-abbauenden Enzyme nach Dialyse gegen $\mathrm{Na}_{3}$-EDTA durch divalente Kationen in verschiedenen Konzentrationen (angegeben als Endkonzentration in
1:10 verd. Plasma). Zahlen: Prozent Verlust an pressorischer Wirkung. 1:10 verd. Plasma). Zahlen: Prozent Verlust an pressorischer Wirkung. partielle Hemmung.- - totale Hemmung. + partielle Reakti
vierung. + + totale Reaktivierung. $O$ keine Reaktivierung

\begin{tabular}{|c|c|c|c|c|c|c|c|}
\hline \multicolumn{2}{|c|}{ Substrat } & \multicolumn{3}{|c|}{$\begin{array}{l}\alpha \text {-L-Aspartyl }{ }^{1}- \\
\text { Angiotensin II } \\
\text { (A) }\end{array}$} & \multicolumn{3}{|c|}{$\begin{array}{c}\alpha-L-\text { Asparaginyl1- } \\
\text { Angiotensin II } \\
\text { (H) }\end{array}$} \\
\hline \multicolumn{2}{|c|}{$\begin{array}{l}\text { pH } \\
\text { Inkubationszeit (Min). }\end{array}$} & $\begin{array}{l}5,6 \\
720\end{array}$ & $\begin{array}{l}6,8 \\
180\end{array}$ & $\begin{array}{l}8,0 \\
180\end{array}$ & $\begin{array}{r}5,6 \\
60\end{array}$ & $\begin{array}{c}7,2 \\
15\end{array}$ & $\begin{array}{l}8,6 \\
15\end{array}$ \\
\hline \multicolumn{2}{|c|}{ Plasma $1: 10$ verdünnt } & 47 & 48 & 50 & 39 & 53 & 45 \\
\hline \multicolumn{2}{|c|}{$\begin{array}{l}\text { Nach Dialyse gegen 0,9proz. } \\
\mathrm{NaCl}\end{array}$} & -1 & $\underline{21}$ & $\underline{25}$ & 0 & 25 & $\underline{23}$ \\
\hline \multicolumn{2}{|c|}{$\begin{array}{l}\text { Nach Dialyse gegen } 0,01 \mathrm{M} \\
\mathrm{Na}_{2} \text { EDTA }\end{array}$} & - & 0 & 0 & 0 & 15 & 12 \\
\hline \multicolumn{8}{|c|}{$\begin{array}{l}\text { Nach anschließender Re- } \\
\text { aktivierung mit }\end{array}$} \\
\hline 025 & $\mathrm{Ca}+$ & $\begin{array}{c}22 \\
+\end{array}$ & $\begin{array}{l}44 \\
++\end{array}$ & $\begin{array}{l}50 \\
++\end{array}$ & $\stackrel{0}{0}$ & $\begin{array}{l}58 \\
++\end{array}$ & $\begin{array}{l}50 \\
++\end{array}$ \\
\hline $2,5 \mathrm{~mm}$ & $\mathrm{Ca}^{++}$ & $\begin{array}{l}47 \\
++\end{array}$ & $\begin{array}{l}45 \\
++\end{array}$ & $\begin{array}{l}50 \\
++\end{array}$ & $\stackrel{0}{0}$ & $\begin{array}{l}56 \\
++\end{array}$ & $\begin{array}{l}50 \\
++\end{array}$ \\
\hline $0,1 \mathrm{~mm}$ & $\mathrm{Mg}^{++}$ & -1 & 0 & $\begin{array}{c}44 \\
++\end{array}$ & $0^{0}$ & $\begin{array}{l}53 \\
++\end{array}$ & 0 \\
\hline $1 \mathrm{~mm}$ & $\mathrm{Mg}^{++}$ & 0 & 5 & $\begin{array}{c}45 \\
++\end{array}$ & 0 & $\begin{array}{c}50 \\
++\end{array}$ & 5 \\
\hline $5 \mu \mathrm{M}$ & $\mathrm{Zn}^{++}$ & $\begin{array}{c}35 \\
+\end{array}$ & 5 & $\begin{array}{c}36 \\
+\end{array}$ & 1 & $\begin{array}{l}40 \\
+\end{array}$ & $\stackrel{24}{(+)}$ \\
\hline $50 \mu \mathrm{M}$ & $\mathrm{Zn}^{++}$ & $\begin{array}{c}45 \\
++\end{array}$ & 6 & $\begin{array}{l}45 \\
++\end{array}$ & $\begin{array}{c}33 \\
++\end{array}$ & $\begin{array}{l}41 \\
+\end{array}$ & $\begin{array}{r}45 \\
++\end{array}$ \\
\hline $500 \mu \mathrm{M}$ & $\mathrm{Zn++}$ & $\begin{array}{c}38 \\
+\end{array}$ & $0^{5}$ & $\begin{array}{l}33 \\
+\end{array}$ & $\begin{array}{r}38 \\
++\end{array}$ & $\begin{array}{l}25 \\
+\end{array}$ & 0 \\
\hline $10 \mu \mathrm{M}$ & $\mathrm{Mn}^{++}$ & 0 & $0^{7}$ & $\begin{array}{l}9 \\
0\end{array}$ & 0 & $\begin{array}{l}41 \\
+\end{array}$ & 0 \\
\hline $100 \mu \mathrm{M}$ & $\mathrm{Mn}^{++}$ & 5 & $0^{7}$ & (O) & 0 & $\begin{array}{c}50 \\
++\end{array}$ & 0 \\
\hline $1 \mu \mathrm{M}$ & $\mathrm{Co}^{++}$ & $0^{7}$ & $\begin{array}{c}30 \\
+\end{array}$ & $\begin{array}{c}33 \\
+\end{array}$ & 0 & $\begin{array}{c}46 \\
++\end{array}$ & 0 \\
\hline $100 \mu \mathrm{M}$ & $\mathrm{Co}^{++}$ & (0) & $\begin{array}{c}47 \\
++\end{array}$ & $\begin{array}{c}50 \\
++\end{array}$ & $0^{4}$ & $\begin{array}{c}46 \\
++\end{array}$ & 5 \\
\hline
\end{tabular}

und $\mathrm{pH} 8,0$ total, bei $\mathrm{pH}$ 5,6 partiell. Dagegen hat $\mathrm{Ca}^{++}$auf die Hemmung der biologischen Inaktivierung von A durch EDTA-Dialyse bei pH 5,6 keinen EinfluB, während $0,25 \mathrm{~mm} \mathrm{Ca}{ }^{++}$bei $\mathrm{pH} 7,2$ und $\mathrm{pH} 8,6$ total reaktivieren.

$\mathrm{Mg}^{++}(0,1 \mathrm{mM})$ reaktiviert die biologische Inaktivierung von $\mathrm{A}$ nur bei $\mathrm{pH} 8,0$, die von $\mathrm{H}$ nur bei $\mathrm{pH} 7,2$. In allen anderen $\mathrm{pH}-$ Bereichen tritt keine Reaktivierung ein.

$\mathrm{Zn}^{++}$reaktiviert die biologische Inaktivierung von $\mathrm{A}$ bei pH 5,6 und pH 8,0 schon in sehr kleinen Konzentrationen ( 5 bis $50 \mu \mathrm{M}$ ) während bei pH 6,8 keine Reaktivierung eintritt. Höhere $\mathrm{Zn}^{++}$-Konzentrationen $(0,5 \mathrm{~mm})$ reaktivieren bei $\mathrm{pH} \mathrm{5,6}$ und $\mathrm{pH} 8,0$ nur partiell. Wahrscheinlich tritt hier bereits eine Hemmung der reaktivierten Enzyme ein, die wir auch bei Anwendung noch höherer $\mathrm{Zn}^{++}$-Konzentrationen in Vorversuchen beobachten konnten.

$\mathrm{Mn}^{++}(10$ bis $100 \mu \mathrm{M})$ reaktiviert nur die biologische Inaktivierung von $\mathrm{H}$ bei $\mathrm{pH} 7,2$ total. Die Reaktivierung des Abbaues von $\mathrm{A}$ bei $\mathrm{pH} 8,0$ (15\%. Inaktivierung) kann nicht als gesichert gelten, da der Fehler der Angiotensin-Bestimmung an der Ratte in Mn-haltigen Lösungen etwas größer war als bei der Testung anderer Metallionen-haltiger Lösungen, die keine Änderung der Genauigkeit des biologischen Tests bedingten:

$\mathrm{Co}^{++}$reaktiviert bei Konzentrationen von 1 bis $100 \mu \mathrm{M}$ die biologische Inaktivierung von $\mathrm{A}$ bei $\mathrm{pH} 6,8$ und $\mathrm{pH} 8,0$ und die von $\mathrm{H}$ bei $\mathrm{pH} 7,2$ total. In anderen pH-Bereichen ist $\mathrm{Co}^{++}$wirkungslos.

Dreitätige Dialyse von Plasma gegen $1 \mathrm{~mm}$ EDTA hemmt die biologische Inaktivierung von $A$ und $\mathrm{H}$ bei $\mathrm{pH}$ 5,6 total. In den anderen $\mathrm{pH}-$ Bereichen tritt nur partielle Hemmung ein. Reaktivierungsversuche mit zweiwertigen Kationen hatten sinngemäß die gleichen Ergebnisse wie nach Dialyse des Plasmas gegen $10 \mathrm{~mm}$ EDTA-Lösung. Die Genauigkeit der Reaktivierungsversuche ist wegen der nur partiellen Hemmung der Angiotensinase im neutralen und alkalischen Bereich allerdings geringer. Die Ergebnisse werden deshalb nicht im einzelnen dargestellt.

\section{Diskussion}

Die Ergebnisse zeigen, daß sowohl $A$ als auch $H$ durch mindestens drei Enzyme des menschlichen Plasmas mit verschiedenen pH-Optima inaktiviert werden. Das jeweilige Wirkungsmaximum liegt im Neutralbereich, doch sind die $\mathrm{pH}$-Optima der $\mathrm{A}$ - und $\mathrm{H}$-spaltenden Enzyme um 0,4 pH-Einheiten voneinander verschieden. Die pH-Optima der biologischen Inaktivierung von A und $\mathrm{H}$ im Sauren sind identisch. Im schwach Alkalischen ergeben sich erneut Unterschiede. Das $\mathrm{pH}-$ Optimum der Inaktivieiung von A liegt bei $\mathrm{pH} 7,6$ bis 8,0 , das von $\mathrm{H}$ weiter im Alkalischen. Die pH-Optima der Freisetzung von Asparagin aus $\mathrm{H}$ bei Substratsättigung liegen bei $\mathrm{pH} 6,2-6,4$ und bei $\mathrm{pH} 7,2$. Nur das Aktivitātsmaximum bei $\mathrm{pH} \quad \overline{7}, 2$ und die enneute Zunahme der Asparagin-Abspaltung im Alkalischen 


\section{PERKIN-ELMER}

\section{GERÄTE FUR DIE KLINISCHE CHEMIE}

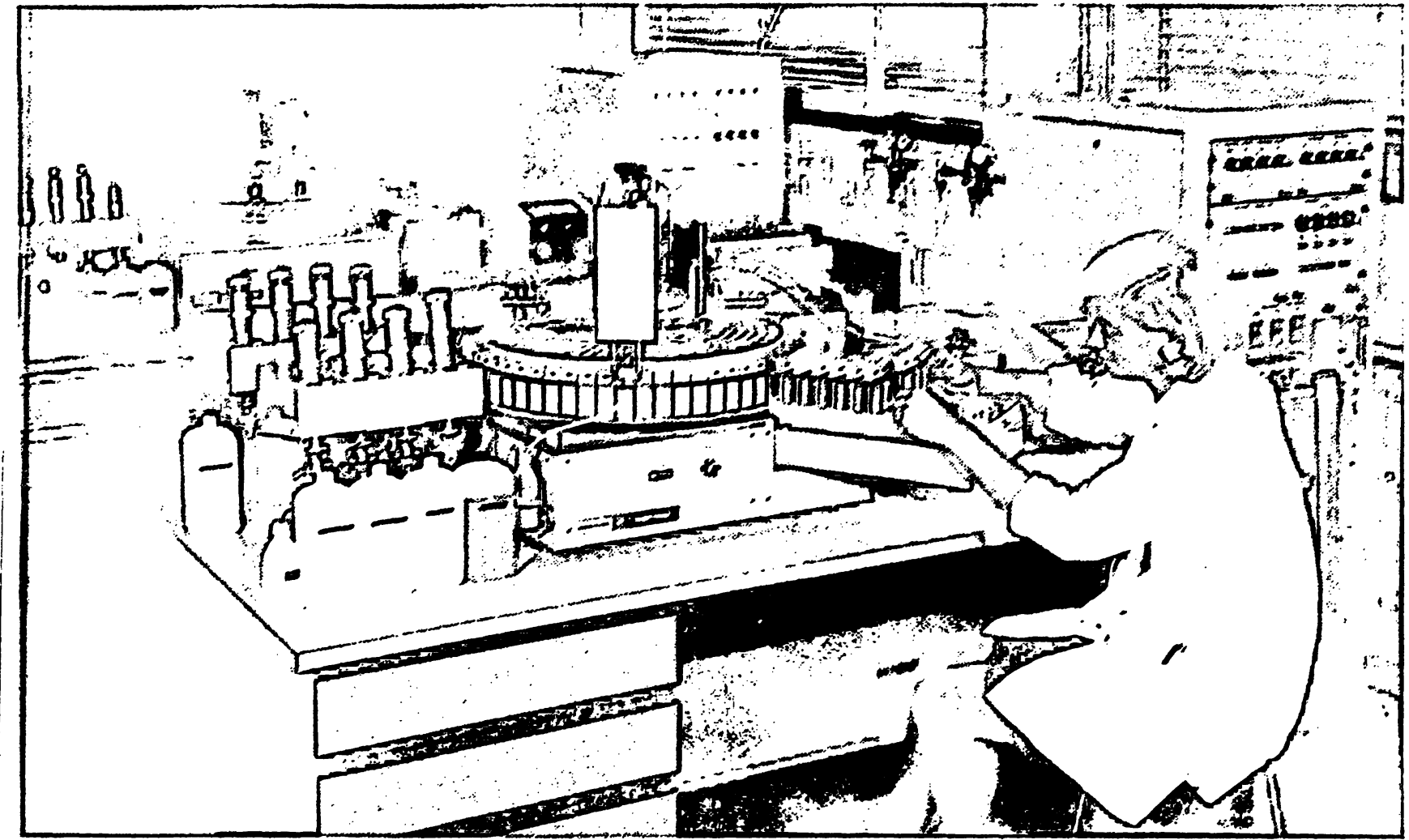

Der PERKIN-ELMER ANALYSENAUTOMAT C4 ist ein Vierkanalgerät mit automatischem Ablauf aller Arbeitsgänge vom Dosieren der Probe über die Reagenz-Zuführung, das Durchführen der Reaktion, das Photometrieren bis zur Auswertung der Messung und dem digitalen Ausdrucken des Ergebnisses. Neben der Automation dieser Arbeitsgănge sind die systembedingte hohe Reproduzierbarkeit und eine direkte, positive Ergebnisidentifizierung hervorstechende Merkmale des Analysenautomaten C 4 .

Das Analysenergebnis wird in Konzentrationseinheiten ausgedruckt. Durch den Wegfall jeglicher Rechenarbeit bei der Ausvertung ergibt sich eine Sicherheit, die bei den bisher üblichen Verfahren unbekannt war.

Beim Analysenautomaten $\mathrm{C} 4$ vird jede Probe in individuelle Reaktionsgefä.Be dosiert. Der Proben- und Reagenzienbedarf ist minimal.

Die Messung wird unter optímalen Eedingungen mit einem Gitterspektrophotometer durchgeflihrt.

In kontinuierlichem Betrieb sind bis zu 120 Analysen je Stunde möglich. Durch die vier voneinander unabhängigen Kanäle können bis zu vier verschiedene Bestimmungen gleichzeitig aus einer Probe durchgeführt werden. Thermostatieierung in der Reaktionszeit, vollständiger Reaktionsablauf vor dem Photometrieren und direkte Messung gegen einen "A.nalysenleerwert" für jede Probe gewährleisten eine hohe Genauigkeit und Reproduzierbarkeit. Das Umrügten auf andere Analysenarten ist unproblematisch und kann rasch durchgeführt werden.

Weitere PERKIN-ELMER-Geräte für die klinische Chemie: Spektrophotometer für den UV-, Sichtbarund IR-Bereich, Fluoreszenzspektrophotometer, Atom-Absorptions-Spektrophotometer, Gas-Chromatographen, Polarimeter und Massen-Spektrometer. 


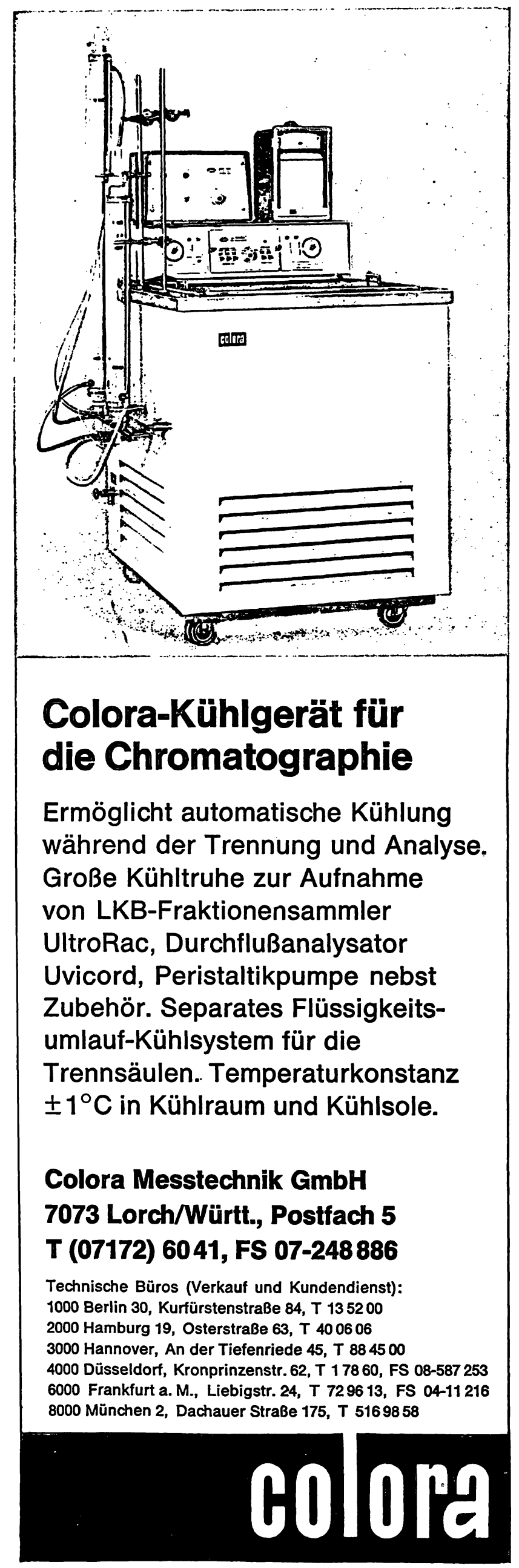

\section{H Me}

für Präzision

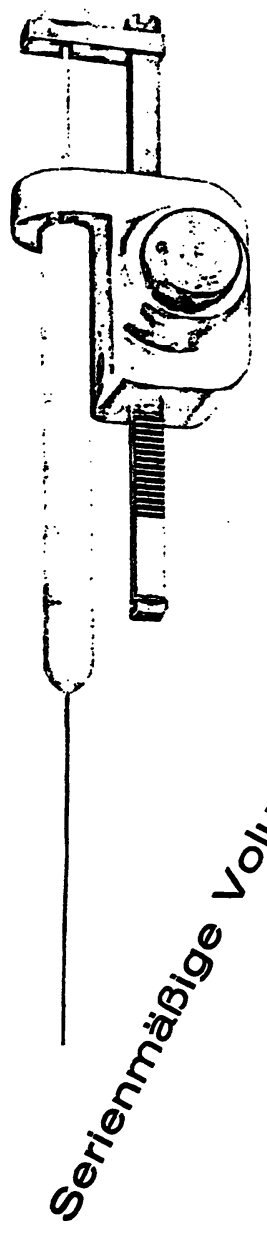

\section{und Qualität}

PB-600

Dosiervorrichtung

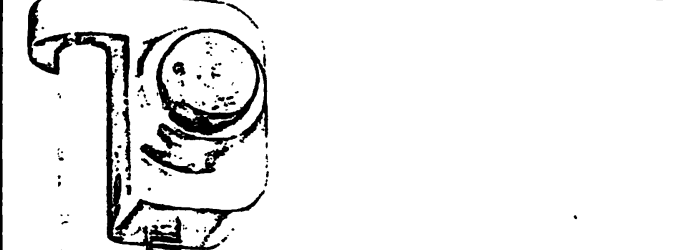

Generalagentur für Europa:

MICROMESURE N.V. Postfach 205 DEN HAAG Holland

Autorisierter Händler für die BRD: GUNTHER SCHMIDT

2 Hamburg 68 Postfach 680104

Bezirksvertretungen:

$\begin{array}{ll}\text { Aachen: } & \text { Fa. Ludwig Mohren OHG } \\ \text { Berlin: } & \text { Fa. K. Marggraf } \\ \text { Bonn: } & \text { Fa. C. Gerhardt } \\ \text { Bremen: } & \text { Fa. H. Jürgens \& Co. } \\ \text { Frankfurt: } & \text { Fa. Willi Fischer \& Co. } \\ \text { Freiburg: } & \text { Fa. Bender \& Hobein } \\ \text { Göttingen: } & \text { Fa. Bodo Schmidt } \\ \text { Hannover: } & \text { Fa. H. Jürgens \& Co. } \\ \text { Karlsruhe: } & \text { Fa. Bender \& Hobein } \\ \text { Kiel: } & \text { Fa. Erich Eydam } \\ \text { München: } & \text { Fa. Bender \& Hobein } \\ \text { Münster: } & \text { Fa. H. Jürgens \& Co. }\end{array}$


korrespondieren mit der pH-Abhängigkeit der biologischen Inaktivierung von $\mathrm{H}$. Das Maximum der Asparagin-Abspaltung bei pH 6,2-6,4 könnte einem Enzym mit geringer Substrataffinität entsprechen, das bei wesentlich kleineren Substratkonzentrationen, wie sie für die Messung der biologischen Inaktivierung verwendet werden, nur wenig Substrat umsetzt. Das bei $\mathrm{pH}$ 5,6 beobachtete pH-Optimum der biologischen Inaktivierung fehlt dagegen in der $\mathrm{pH}$-Optimum-Kurve der Aminopeptidase-Aktivität. Dieser Befund könnte die Vermutung von Khatrallah und Mitarbeiter (G) stützen, daß die „saure Angiotensinase“, über die schon Pickens und Mitarbeiter (10) berichteten, eine Endopeptidase ist. Pickens und Mitarbeiter (10) und KhaIRALLAH und Mitarbeiter (6) fanden, daß die Angitensinase-Aktivität in diesem Bereich mit Diisopropylfluorophosphat hemmbar ist. Sie schlossen daraus auf eine dem . Chymotrypsin ähnliche Wirkungsweise. Khatrallah und Mitarbeiter (6) konnten die „saure ATase" kürzlich durch Ammoniumsulfatfraktionierung und Chromatographie über Sephadex G-75 von der Angiotensinase A. trennen. Sie fanden ihr Wirkungsoptimum zwischen pH 5,8 und 6,0. Das Enzym spaltet sowohl $A$ als auch $H$, letzteres doppelt so schnell wie A. Aber auch Angiotensin-Analoge wie $\beta$-Aspartyl ${ }^{1-}$ Angiotensin $I I$ und Succinyl1-Angiotensin II, die von der Angiotensinase A nicht angegriffen werden, kann das Enzym inaktivieren. KhaIrallaH und Mitarbeiter (6) fanden, daß das Enzym durch Dialyse gegen EDTA inaktiviert und durch $\mathrm{Ca}^{++}, \mathrm{Mn}^{++}, \mathrm{Mg}^{++}$und $\mathrm{Co}^{++}$ nicht reaktiviert wird. Sie schlossen daraus, daß die Inaktivierung durch EDTA nicht durch Chelatbildung mit einem metallischen Cofaktor, sondern durch Denaturierung des Enzyms bedingt sei. Offenbar haben KHAIRALLAH und Mitarbeiter (6) ihre Reaktivierungsversuche nur mit dem Substrat $\mathrm{H}$ durchgeführt. Ihre Befunde stimmen dann mit unseren vollkommen überein (siehe Tab. 1). Das von KhaIrallay und Mitarbeiter als potentieller Reaktivator nicht getestete $\mathrm{Zn}^{++}$ vermag jedoch die EDTA-Hemmung schon in sehr kleinen Konzentrationen vollständig aufzuheben (Tab. 1). Die Hemmung des Abbaues von $A$ durch EDTA bei pH 5,6 wird dagegen sowohl durch $\mathrm{Zn}^{++}$als auch durch $\mathrm{Ca}^{++}$aufgehoben. $\mathrm{Da}$ die Stabilitätskonstante des EDTA-Calcium-Chelates bei $\mathrm{pH} \mathrm{5,6}$ wesentlich kleiner ist als im Neutralbereich (13), und da die "saure ATase“ schon durch Dialyse gegen 0,9proz. NaCl-Lösung bzw. durch Zusatz kleiner EDTA-Konzentrationen zum Reaktionsgemisch (Abb. 3 und 4) gehemmt wird, ist es unwahrscheinlich, $\mathrm{daB} \mathrm{Ca}^{++}$der natürliche Cofaktor der A-spaltenden "sauren ATase" ist. Die Stabilität des EDTA-Zink-Chelates ist um etwa fünf Zehnerpotenzen größer als die des EDTA-Calcium-Chelates (13). Daher ist es wahrscheinlich, daß $\mathrm{Zn}^{++}$oder ein Metallion mit ähnlicher Affinität zu EDTA der natürliche Aktivator der "sauren ATase“ ist. Die Inaktivierbarkeit des Enzyms durch Dialyse gegen 0,9proz. $\mathrm{NaCl}-L$ ösung spricht außerdem gegen eine feste Bindung dieses Metallions an das Enzymprotein. Aus der
Aktivierbarkeit der A-spaltenden Enzymaktivität durch $\mathrm{Ca}^{++}$kann der Schluß gezogen werden, daß die „saure ATase" aus zwei Enzymen besteht, die einerseits $H$ (und $A$ ?), andererseits nur $A$ inaktivieren.

Bei den "neutralen ATasen" findet sich weitgehende Úbereinstimmung zwischen den Ergebnissen von Khatrallah und Mitarbeiter (6) und den unseren. Khatrallah und Mitarbeiter konnten die durch Ammoniumsulfatfraktionierung von der ,saurenATase“ (Angiotensinase B) getrennte Angiotensinase A $(5 ; 6)$ durch weitere Fraktionierung in zwei Enzyme auftrennen, deren eines (Angiotensinase $A$ 1) nur $\mathrm{H}$ inaktiviert, während die Angiotensinase A 2, die bei 2,35M $\left(\mathrm{NH}_{4}\right)_{2} \mathrm{SO}_{4}$-Konzentration präzipitiert, nur $\mathrm{A}$ inaktiviert. Beide werden durch Dialyse gegen EDTA ( $3 \mathrm{~mm}$ ) vollständig gehemmt und durch $\mathrm{Ca}^{++}$reaktiviert. Das pH-Optimum der ATase A 1 liegt bei $\mathrm{pH} 7,4$, das der ATase 2 bei $\mathrm{pH}$ 6,8. Die von uns gefundenen unterschiedlichen $\mathrm{pH}$-Optima der Inaktivierung von $\mathrm{A}$ und $\mathrm{H}$ im neutralen Bereich sind also nicht, was man vermuten könnte, durch methodische Ungenauigkeit bedingt, sondern zeigen die Existenz zweier Enzyme an. Unterschiede finden sich auch in der Reaktivierbarkeit der nur durch höhere EDTA-Konzentrationen hemmbaren Enzyme (Abb. 3 und 4, Tab. 1) mit zweiwertigen Kationen. Während die durch EDTA gehemmte Spaltung von $\mathrm{A}$ bei $\mathrm{pH}$ 6,8 nur durch $\mathrm{Ca}^{++}$und $\mathrm{Co}^{++}$reaktiviert wird, wird die von $\mathrm{H}$ bei $\mathrm{pH} 7,2$ durch $\mathrm{Ca}^{++}$, $\mathrm{Mg}^{++}, \mathrm{Mn}^{++}$und $\mathrm{Co}^{++}$reaktiviert. Hier ergeben sich andererseits Unterschiede gegenüber den Befunden von KKaIRALlah und Mitarbeitern (6), die eine totale Reaktivierung des durch EDTA gehemmten Komplexes Angiotensinase A nur mit $1 \mathrm{~mm} \mathrm{Ca}{ }^{++}$und eine partielle mit $1 \mathrm{mM} \mathrm{Mn}^{++}$und $\mathrm{Co}^{++}$fanden. $1 \mathrm{mMMg}^{++}$war unwirksam. Die Autoren geben allerdings nicht an, mit welchem Substrat (A oder $\mathrm{H}$ ) diese Untersuchungen durchgeführt wurden. Manche Unterschiede zwischen unseren Ergebnissen und denen von KhaIrallaH und Mitarbeitern (6) könnten aber durch unterschiedliche Konzentrationen der Metallionen erklärt werden. Wir haben in Vorversuchen gefunden, da $\beta$ bereits $0,5 \mathrm{~mm}$ $\mathrm{Mn}^{++}$oder $\mathrm{Co}^{++}$die neutralen ATasen partiell hemmen können, während kleinere Konzentrationen total reaktivierten. Diese partielle Hemmung kann, wenn nur eine Metallionenkonzentration geprüft wird, auch als partielle Reaktivierung interpretiert werden.

Die im schwach alkalischen Bereich $\mathrm{A}$ und $\mathrm{H}$ inaktivierenden Enzyme sind hinsichtlich ihrer $\mathrm{pH}$-Abhängigkeit und Reaktivierbarkeit durch Metallionen nach Dialyse gegen EDTA eindeutig unterschieden. Die $\mathrm{H}$ inaktivierende Enzymaktivität oberhalb pH 8,0 ist erst mit höheren EDTA-Konzentrationen als das A-spaltende Enzym (pH-Optimum 7,6-8,0) hemmbar (Abb. 3 und 4, Tab. 1). Es wird durch $\mathrm{Ca}^{++}$und $\mathrm{Zn}^{++}$reaktiviert, während das A-spaltende Enzym durch $\mathrm{Ca}^{++}$, $\mathrm{Mg}^{++}, \mathrm{Zn}^{++}$und $\mathrm{Co}^{++}$total reaktiviert wird. Auch in diesem Bereich können unsere Untersuchungen eine Beteiligung mehrerer Enzyme an der Inaktivierung des jeweiligen Substrats nicht ausschließen. Der Vergleich 
der pH-Abhängigkeit der biologischen Inaktivierung von $\mathrm{H}$ und der Asparagin-Abspaltung aus $\mathrm{H}$ (Abb. 2) läßt den Schluß zu, daß die ,neutrale“ und die ,alkalische" $\mathrm{H}$-spaltende ATase Aminopeptidasen sind. Unsere Ergebnisse und die von Krairallah und Mitarbeitern ( 6 ) bestätigen einander in vieler Hinsicht. Widersprüche können zum Teil durch unterschiedliche Versuchsanordnungen erklärt werden. Die Heterogenität der „Angiotensinasen" ist bemerkenswert. Sie sollte bei zukünftigen Untersuchungen mehr berücksichtigt werden.

Wir danken Herrn Prof. Dr. F. Gross von der CIBA-AG in Basel für die freundliche Überlassung von $\alpha$-L-Ạngiotensin II und $\alpha$-L-Angiotensin II-Amid.

Der Deutschen Forschungsgemeinschaft danken wir für die Unterstützung dieser Arbeit.

\section{Literatur}

1. Regoli, D., B. Riniker und H. Brunner, Biochem. Pharmacol. 12, 637 (1963). - 2. Nagatsu, I., L. Gillespie, J. E. Folk und G. G. GlenNer, Biochem. Pharmacol. 14, 721 (1965). - 3. Nagatsu, I., L. Gillespie, J. M. George, J. E. Folk und G. G. GlenNer, Biochem. Pharmacol. 14, 853 (1965). - 4. GlenNER, G. G., P. J. McMillan und J. E. Folk, A Nature (London) 194, 867 (1962). - 5. Khatrallah, P. A., F. M. Bumpus, I. H. Page und R. R. SMeBY, Science Washington 140, 672 (1963). - 6. Khatrallah, P. A. und I. H. Page, Biochemical Medicine 1, 1 (1967). - 7. v. Goldacker, I. U. und W. OelKers, diese Z. 7,
250 (1969) vorstehend. - 8. HeLmer, O. M. und W. E. Judson, Circulation 27, 1050 (1963). - 9. Brown, J. J., D. L. Davies, A. F. LeVER, J. I. S. Robertson und M. TREe, Biochem. J. 93, 594 (1964). - 10. Pickens, P. T., F. M. Bumpus, A. M. Lloyd, R. R. SMEBY und I. H. PAGE, Circul. Res. 17, 438 (1965). - 11. Oelkers, W. und I. U. v. Goldacker, Klin. Wschr. 45, 649 (1967). - 12. Hickler, R. B., D. P. LAULER und G. W. ThorN, J. Clin. Invest. 42, 635 (1963). - 13. Chabarek, S. und A. E. MartelL, Organic Sequestring Agents. P. 588. John Wiley \& Sons, Inc. Publ., New York (1959).

cand. med. M. Zwanzig und Dr. med. W. Oelkers II. Medizinische Klinik und Poliklinik 1 Berlin 45

Hindenburg-Damm 30 